\title{
The crucial role of a phosphatase in insulin resistance and obesity
}

\author{
Anath Shalev \\ Diabetes Branch, National Institute of Diabetes and Digestive and Kidney Diseases, National Institutes of Health, Bethesda, Maryland 20892, USA; \\ Email: anaths@intra.niddk.nih.gov
}

Insulin binding to its receptor leads to autophosphorylation and activation of the tyrosine kinase of the $\beta$-subunit. This step initiates a cascade of substrate phosphorylation events which confer the metabolic as well as the mitogenic effects of insulin. Research has therefore focused mainly on the kinases involved in these phosphorylation cascades. However, a number of phosphatases are able to counteract these processes by dephosphorylating the insulin receptor (IR) and thus may play an integral role in insulin signaling. One of these phosphatases is the protein tyrosine phosphatase1B (PTP-1B). Recently, Elchebly et al. (1) created a knockout mouse by disrupting the PTP-1B gene and found that the homozygote mice (PTP-1B-/-) that are completely deficient in that enzyme show increased IR phosphorylation, increased sensitivity to insulin and are resistant to diet-induced obesity.

Interestingly, the PTP-1B-/- mice grow and develop normally, are fertile and show no macroscopic or histological differences compared with wild-type mice $(\mathrm{PTP}-1 \mathrm{~B}+/+)$. In the fasted state, they were also metabolically normal, with glucose and insulin levels comparable to wild-type mice. However, when fed ad libitum, glucose and insulin plasma concentrations were significantly lower in PTP-1B-/- compared with PTP$1 \mathrm{~B}+/+$ mice, suggesting an increased insulin sensitivity in the knockout mice. Oral glucose tolerance tests (OGTTs) and intraperitoneal insulin tolerance tests (ITTs) confirmed this hypothesis, as the mutant mice showed more rapid glucose clearance and prolonged hypoglycemia respectively. These findings were associated with an increased and prolonged phosphorylation of the IR in liver and muscle (consistent with the lack of dephosphorylation due to absent PTP-1B) while the amount of IR expression was the same in PTP-1B-/and PTP $-1 \mathrm{~B}+/+$ mice. Elchebly and colleagues further showed that the increased phosphorylation of the IR is in fact functional as well as specific. Insulin-induced phosphorylation of the insulin receptor substrate-1 (IRS-1), a major factor in insulin signaling, was enhanced in muscle of PTP-1B-/- mice, reflecting an increased activity of the IR tyrosine kinase. On the other hand, epidermal growth factor receptor tyrosine kinase was found to be equally active in PTP-1B-/- and PTP$1+/+$ mice, suggesting that disruption of the PTP-1B gene increased specifically the tyrosine kinase activity of the IR. Substrate specificity of the PTP-1B is thought to be due to specific interactions between distinct amino acid residues in the substrate and the enzyme (2).

The phenotype of the PTP-1B-/- mice described above can be explained by the targeted disruption of the PTP-1B with reduced inactivation of the IR, leading to increased IR phosphorylation in muscle and liver, enhanced tyrosine kinase activity and phosphorylation of IRS-1 and consecutive stimulation of the insulin signaling pathway resulting in increased insulin sensitivity. However, Elchebly and co-workers discovered an additional unexpected feature in this knockout mouse. When the mice were fed a high fat diet $(50 \%$ of calories from fat) in order to investigate the effect of the PTP-1B disruption in obesity-induced insulin resistance, they noticed that the PTP-1B-/- mice failed to gain weight to the same extent as wild-type mice. Interestingly, this was not due to decreased amounts of food intake since there was no difference in the amount of calories consumed. While wild-type animals fed the high fat diet were found to have markedly increased glucose and insulin levels, PTP-1B-/- mice continued to have normal low glucose and insulin concentrations. Studies looking at OGTTs, ITTs and IR phosphorylation in animals fed the high fat diet revealed an obesity-induced insulin resistance and decreased IR phosphorylation in the wild-type mice, whereas the knockout mice seemed to be protected. This resulted in an even greater difference in insulin sensitivity and IR phosphorylation between PTP-1B-/- and wild-type mice.

The mechanism for this resistance to diet-induced obesity is not known although the authors speculate that fat metabolism is affected in the mutant mice since they found reduced plasma concentrations of triglycerides in the PTP-1B-/- compared with the PTP-1B $+/+$ mice. Interestingly, IR phosphorylation in adipose tissue seems to be unaffected by the disruption of PTP-1B, suggesting a different, probably indirect, mechanism for this effect on fat deposition.

Lack of PTP-1B seems to confer both increased insulin sensitivity and resistance to obesity and thus makes this enzyme a potential therapeutic target $(1,3)$. PTP-1B is a member of the family of eukaryotic protein tyrosine phosphatases (PTPs), reviewed in (4). Based upon their cellular localization, the phosphatases are divided into membrane-bound (e.g. leucocyte common antigen-related) and cytoplasmic enzymes (e.g. PTP-1B). There is also a third group called dual specificity 
phosphatases due to their ability to remove phosphates from threonine and serine residues in addition to their tyrosine phosphatase activity. Vanadate has been known for a long time to be a non-specific inhibitor of PTPs and to mimic the effects of insulin (for review see 5), but the toxicity and non-selective nature of vanadate makes it unattractive as a therapeutic agent, and the question of which PTP is involved in the insulinomimetic action remains unanswered. Over the last years data have begun to accumulate that PTP-1B plays a major role as a negative regulator of insulin signaling (reviewed by Byon et al. (6)). Therefore several groups started the search for PTP-1B specific inhibitors. In fact, almost simultaneously with the publication of the PTP-1B knockout mouse, Tiang and collaborators (7) described a class of potent and highly selective inhibitors of PTP1B. Based upon the crystallographic structure of PTP1B, Tiang et al. designed these inhibitors as a chemical substance class of aryl difluorophosphonates. These substances still have to be tested in vivo for their efficacy and potential toxicity but they may be potential therapeutic agents for diabetes and obesity. Since unopposed phosphorylation of the IR and stimulation of the insulin transduction pathway could also induce the mitogenic effects of insulin, blocking PTP-1B may potentially cause tumorigenesis. On the other hand, there has been no evidence of an increased cancer rate after 2 years of observation of the PTP-1B-/- mice $(1,3)$.

Besides the therapeutic implications, the PTP-1B mouse model raises many physiological questions. If loss of PTP-1B activity protects against insulin resistance and obesity, it is likely that PTP-1B plays an important role in the pathogenesis of diabetes and weight gain. Interestingly, a transgenic mouse model of non-insulin-dependent diabetes mellitus due to deficiency of the G-protein subunit $\mathrm{G}_{\mathrm{i} \alpha 2}$ shows markedly increased PTP activity and PTP-1B expression (8). It would be tempting to disrupt the PTP-1B gene in this model and see whether the mice could be rescued from the diabetic phenotype. Whether PTP-1B is overactive in other rodent models or humans with insulin resistance, diabetes and obesity is unknown. It certainly would be interesting to know whether there are any mutations or polymorphisms in the human PTP-1B gene that are responsible for or provide increased susceptibility to diabetes and weight gain. Furthermore, it is not known how PTP-1B is regulated in the normal state. For instance, is it under the control of insulin or leptin? In any event, these recent findings on PTP-1B have demonstrated that phosphatases can be a counterpoint to the protein tyrosine kinases in insulin signaling and may play crucial roles in diabetes and obesity as well as serve as targets for treatment.

\section{References}

1 Elchebly M, Payette P, Michaliszyn E, Cromlish W, Collins S, Loy AL et al. Increased insulin sensitivity and obesity resistance in mice lacking the protein tyrosine phosphatase-1B gene. Science $19992831544-1547$.

2 Sarmiento M, Zhao Y, Gordon SJ \& Zhang Z-Y. Molecular basis for substrate specificity of protein-tyrosine phosphatase 1B. Journal of Biological Chemistry $199827326368-26374$.

3 Ferber D. New clues found to diabetes and obesity. Science 1999 283 1424-1425.

4 Huijsduijnen RHV. Protein tyrosine phosphatases: counting trees in the forest. Gene $19982251-8$.

5 Morinville A, Maysinger D \& Shaver A. From Vanadis to Atropos: vanadium compounds as pharmacological tools in cell death signalling. Trends in Pharmacological Science $199819452-460$.

6 Byon JC, Kusari AB \& Kusari J. Protein-tyrosine phosphatase-1B acts as a negative regulator of insulin signal transduction. Molecular and Cellular Biochemistry 1998182 101-108.

7 Tiang M, Keng Y-F, Shen K, Wu L, Lawrence DS \& Zhang Z-Y. Potent and highly selective inhibitors of the protein tyrosine phosphatase 1B. Biochemistry 199938 3793-3803.

8 Moxham CM \& Malbon CC. Insulin action impaired by deficiency of the G-protein subunit Gi $\alpha 2$. Nature 1996379 840-844. 